\title{
Ottawa Hospital Patient Safety Study: incidence and timing of adverse events in patients admitted to a Canadian teaching hospital
}

\author{
Alan J. Forster, Tim R. Asmis, Heather D. Clark, Ghiath Al Saied, Catherine C. Code, \\ Sharon C. Caughey, Kevin Baker, James Watters, Jim Worthington, Carl van Walraven
}

Abstract

Background: Adverse events are poor patient outcomes that are due to medical care. Studies of hospital patients have demonstrated that adverse events are common, but few data describe the timing of them in relation to hospital admission. We evaluated characteristics of adverse events affecting patients admitted to a Canadian teaching hospital, paying particular attention to timing.

Methods: We randomly selected 502 adults admitted to the Ottawa Hospital for acute care of nonpsychiatric illnesses over a 1 -year period. Charts were reviewed in 2 stages. If an adverse event was judged to have occurred, a physician determined whether it occurred before or during the index hospitalization. The reviewer also rated the preventability, severity and type of each adverse event.

Results: Of the 64 patients with an adverse event (incidence $12.7 \%$, 95\% confidence interval [Cl] 10.1\%-16.0\%), 24 had a preventable event $(4.8 \%, 95 \% \mathrm{Cl} 3.2 \%-7.0 \%)$, and $3(0.6 \%$, $95 \% \mathrm{Cl} 0.2 \%-1.7 \%$ ) died because of an adverse event. Most adverse events were due to drug treatment, operative complications or nosocomial infections. Of the 64 patients, 39 (61\%, $95 \% \mathrm{Cl} 49 \%-72 \%$ ) experienced the adverse event before the index hospitalization.

Interpretation: Adverse events were common in this study. However, only one-third were deemed avoidable, and most occurred before the hospitalization. Interventions to improve safety must address ambulatory care as well as hospitalbased care.

CMAJ 2004;170(8):1235-40

$\mathrm{P}$ atient safety has become an area of interest in Canada and in many other countries. This is partly because international research has demonstrated that adverse events, or poor outcomes caused by medical care, are common. Two US studies identified adverse events in $2.5 \%{ }^{1}$ to $3.7 \%^{2}$ of hospitalizations, while British and Australian studies found rates of $10.8 \%{ }^{3}$ and $16.6 \%,{ }^{4}$ respectively.

One aspect of such studies that is often overlooked is the timing of the adverse event with respect to the hospitalization. ${ }^{5}$ Except for the British study, ${ }^{3}$ all previous studies included adverse events that occurred before the hospitalization under review as long as the adverse event was discovered during the hospitalization. ${ }^{1,2,4}$ Such "prehospital" events constituted between $38 \%{ }^{2}$ and $49 \%$ of all adverse events in these studies. Yet, although the overall incidence of adverse events has been widely publicized, most of the attention on improving safety has focused on hospital care.

We performed this study to determine the incidence, preventability, severity, type and timing of adverse events affecting patients in a Canadian teaching hospital. In addition, we evaluated differences in adverse events occurring before and during hospitalization. This information would confirm previous observations that many adverse events occur before admission. Importantly, we felt that a more thorough evaluation of the timing and location of adverse events could lead to more rational interventions to improve quality and safety.

\section{Methods}

The study took place in the Ottawa Hospital, a multicampus teaching hospital, and was approved by the Ottawa Hospital Research Ethics Board. We chose a random sample of 502 adult, nonpsychiatric hospitalizations from 2 acute-care facilities over a 1 -year period. The sample constituted $1.3 \%$ of all hospitalizations and was stratified by facility and admitting service.

As in previous studies, we used a 2 -stage chart-review process (Appendix 1) to identify adverse events. ${ }^{1-4}$ In brief, in stage 1 a nurse reviewed charts to identify hospitalizations meeting at least 1 of 16 screening criteria. The criteria were identical to those used in previous studies and were selected to identify admissions in which an adverse event could have occurred. The nurse-reviewer had received a 1-hour training session on patient-safety issues, background literature and chart-review methods. In addition, the nurse and the principal investigator reviewed a training sample of 50 charts independently and discussed discrepancies in coding.

In stage 2, the charts rated "screen-positive" by the nursereviewer were reviewed by 1 of 8 physicians (surgeons, internists, obstetricians and an emergency physician) to determine whether an adverse event had occurred. The physicians had received a 2 hour training session with the same framework as the nurse's training session. In addition, the physicians and the principal investigator reviewed a training sample of 5 charts independently and discussed discrepancies in coding. The physicians used a 6- 
point scale to rate their confidence that an adverse outcome was due to medical care. A score of 4 or above indicated that the outcome was an adverse event. All hospitalizations that were rated positive for an adverse event and a sample of negative-rated charts were reviewed again by another physician.

Adverse events were rated for preventability, severity, type, timing and location. A preventable adverse event was one that, on the basis of implicit judgement, was felt to be due to an error in management. Severe adverse events led to permanent disability or death. The type was classified as adverse drug event, operative complication, nosocomial infection, diagnostic error (an indicated test was not ordered or a significant test result was misinterpreted) or system problem (inadequate communication, inadequate training or supervision of doctors, or inadequate functioning of hospital services). Types of adverse events were not mutually exclusive; for example, a patient with a postoperative wound infection was classified as having both a nosocomial infection and an operative injury.

As in previous studies, ${ }^{1,2,4}$ we included adverse events that occurred before or during the index hospitalization as long as the event was first discovered during that hospitalization. We did not include adverse events that developed after the index hospitalization. ${ }^{1,2} \mathrm{We}$ determined whether "prehospital" adverse events occurred during ambulatory care, during an emergency department visit or during a previous hospitalization. "Ambulatory care" consisted of treatment in physician offices, in the patient's home or in a nursing home. If an event occurred in the emergency department and the patient was directly admitted, then the event location was coded as "in-hospital." However, if the patient was sent home from the emergency department and an adverse event required subsequent admission to hospital, the adverse event was classified as prehospital.

The unit of analysis was the hospitalization. We calculated event rates per 100 hospitalizations. We used the Wilson Score method to calculate $95 \%$ confidence intervals (CIs). We analyzed 3 simple variables as potential risk factors for the occurrence of adverse events (patient age [in quartiles], admission status ["elective" if the chart contained a completed "request-for-admission" form] and admitting service [medicine, surgery or obstetrics and gynecology]).

The $\chi^{2}$ test was used to compare event risk between strata and the $\chi^{2}$ test for trend to compare event risk between ranked strata. We determined independent predictors of in-hospital adverse events using a multivariate logistic regression model. Variables were entered into the model if they were significant in univariate testing at $p<0.2$.

Table 1: Types of adverse events in $\mathbf{5 0 2}$ randomly selected hospitalizations in 2 campuses of a Canadian teaching hospital

\begin{tabular}{|c|c|c|c|c|c|}
\hline \multirow{3}{*}{$\frac{\text { Type of adverse event }}{\text { Adverse drug event* }}$} & \multicolumn{5}{|c|}{ No. (and \%) of adverse events } \\
\hline & Preventable & In-hospital & Prehospital & Severe & All \\
\hline & $16 \quad(67)$ & $10 \quad(40)$ & $22 \quad(56)$ & $4 \quad(33)$ & 32 (50) \\
\hline Operative complication & $3 \quad(12)$ & $10 \quad(40)$ & $10 \quad(26)$ & $4 \quad(33)$ & $20 \quad(31)$ \\
\hline Nosocomial infection & (8) & $8 \quad(32)$ & $4(10)$ & $2(17)$ & 12 (19) \\
\hline Diagnostic error & $6 \quad(25)$ & $2 \quad(8)$ & $4(10)$ & 3 (25) & $6 \quad(9)$ \\
\hline System problem & $5 \quad(21)$ & $(8)$ & $3 \quad(8)$ & (8) & $(8)$ \\
\hline Procedure injury & $0 \quad(0)$ & (4) & $4 \quad(10)$ & $2(17)$ & $(8)$ \\
\hline Anesthetic injury & $(0)$ & $(4)$ & $(0)$ & $(0)$ & $(2)$ \\
\hline Obstetric injury & $(0)$ & $(0)$ & (3) & $(0)$ & (2) \\
\hline All & $24(100)$ & $25(100)$ & 39 (100) & 12 (100) & $64(100)$ \\
\hline
\end{tabular}

*Defined as an adverse event caused by medication use.

\section{Results}

We reviewed all the charts for the 502 randomly selected hospitalizations. At least 1 of the screening criteria was present in $312(62.2 \%)$ of the charts, and adverse events (Appendix 2) were identified in 64 charts (incidence $12.7 \%$, $95 \%$ CI $10.1 \%-16.0 \%)$.

Of the 502 patients, 24 had a preventable adverse event $(4.8 \%, 95 \%$ CI $3.2 \%-7.0 \%), 3(0.6 \%, 95 \%$ CI $0.2 \%-$ $1.7 \%)$ died because of an adverse event, $9(1.8 \%, 95 \%$ CI $0.4 \%-3.3 \%)$ had permanent disabilities, and $52(10.4 \%$, $95 \%$ CI $8.0 \%-13.3 \%$ ) had temporary disabilities; none of the deaths was considered preventable, whereas 5 of the permanent disabilities were. Adverse events were most commonly adverse drug events (50\%), surgical complications $(31 \%)$ or nosocomial infections (19\%) (Table 1).

Only 25 of the patients experienced their adverse events after admission to hospital $(5.0 \%$, 95\% CI 3.1\%-6.9\%). The remaining 39 (7.8\%, 95\% CI 5.7\%-10.4\%) experienced them before the index hospitalization. Similar proportions of prehospital and in-hospital adverse events were preventable (32\% and $40 \%$, respectively; $p=0.5$ ) or severe (18\% and 20\%, respectively; $p=0.8$ ). Except for operative events, the types of prehospital adverse events were similar to the types of in-hospital adverse events (Table 1).

Of the 39 prehospital adverse events, 20 (31\% of all adverse events) occurred during ambulatory care, 16 (25\% of all adverse events) during a previous hospitalization and 3 (5\% of all adverse events) during a previous emergency department visit. Of the 20 prehospital adverse events that occurred during ambulatory care, 18 (90\%) were adverse drug events, and 9 (45\%) were preventable. Of the 16 adverse events that occurred during previous hospitalizations, $10(62 \%)$ were operative complications, 3 (19\%) were adverse drug events, 3 (19\%) were nosocomial infections, and $4(25 \%)$ were preventable. All 3 adverse events that occurred during previous emergency department visits were due to diagnostic errors and were preventable. The difference in the proportion of adverse events that were judged to be preventable in each location was significant $(p<0.05)$.

Table 2 presents the incidence of different types of adverse events by patient age group, admitting service and admission status. Overall risk of adverse events was significantly associated with patient age $\left(p<0.001\right.$ by the $\chi^{2}$ test for trend), admitting service $(p=0.001)$ and admission status $(p=0.04)$. When only in-hospital adverse events were considered, however, the risk remained significantly associated with age $(p=0.004)$ but was no longer associated with admission status and was less strongly associated with admitting service $(p=0.03)$. After controlling for patient age with a multivari- 
ate logistic model, we found that admitting service was not significantly associated with risk of in-hospital adverse events, although the total number of adverse events was small. Risk of adverse events was the same in both facilities.

\section{Interpretation}

We found that $12.7 \%$ of hospitalizations in a Canadian teaching hospital were associated with an adverse event and that $38 \%$ of all of the adverse events were preventable. We also determined that $61 \%$ of the events occurred before the patient was admitted to the facility. The types and preventability of prehospital adverse events were similar to those of in-hospital events. However, prehospital events that occurred in the ambulatory setting were almost always adverse drug events and were often preventable, whereas those related to previous hospitalizations were more often surgical complications and less often preventable.

Although we used similar methods, comparisons between our study and previous ones need to be made cautiously owing to subtle differences in definitions and reviewer behaviour and international differences in charting practices. ${ }^{5,6}$ However, prehospital adverse events were less common in the US studies than in our study and the Australian one. This could be due to differences in the availability of primary health care: since greater proportions of the population in Canada and Australia are eligible for primary health care, it is possible that greater proportions receive medical treatment in the community. Alternatively, treatments may be monitored and followed more closely in the United States, which would make it less likely that complications would lead to hospitalization. The higher proportion of prehospital adverse events in Canada could also be due to the fact that the study hospital is a referral centre.

Our study has both strengths and limitations. We used standard methods, evaluated more than 1 institution and enrolled appropriate specialists to conduct the chart reviews. However, the 2 facilities that we evaluated were teaching hospitals and in the same city. Current methods for detecting adverse events are hampered by being retrospective and based on chart review. This bias probably results in a conservative rate estimate. Determinations of adverse events are based on implicit criteria and are only moderately reliable. ${ }^{7}$ We tried to address this limitation by presenting all of our adverse events, so that readers could make their own judgements about validity.

In summary, adverse events are relatively common. Most are the consequence of therapies that are provided correctly but have inherent risks. However, many adverse events are potentially preventable. Therefore, increased efforts must be made to reduce their incidence. The higher rate of prehospital adverse events needs to be confirmed. A larger, multicentre Canadian study is underway and may help shed light on this intriguing finding. Regardless, it is clear that quality-improvement efforts must address ambulatory care as well hospital care.

\section{This article has been peer reviewed.}

From the Ottawa Health Research Institute (Forster, Clark, Watters, van Walraven); the Departments of Medicine (Forster, Asmis, Clark, Code, van Walraven), Surgery (Al Saied, Watters) and Obstetrics and Gynecology (Caughey, Baker), University of Ottawa, Ottawa, Ont.; the Department of Emergency Medicine, Ottawa Hospital (Worthington), Ottawa, Ont.; and the Institute for Clinical Evaluative Sciences (van Walraven), Toronto, Ont.

Competing interests: None declared.

Contributors: Alan Forster conceived and designed the study and, with Carl van Walraven, interpreted the data. Alan Forster analyzed the data and drafted the article. All of the authors acquired data, revised the draft critically for important intellectual content and approved the version submitted for publication.

Acknowledgements: This research was supported by a research grant from the Ottawa Hospital and the Ottawa Internists Research Group. Carl van Walraven is an Ontario Ministry of Health Career Scientist.

Table 2: Incidence of adverse events, stratified by patient and hospitalization characteristics

\begin{tabular}{|c|c|c|c|c|c|c|}
\hline \multirow[b]{2}{*}{ Characteristic } & \multirow{2}{*}{$\begin{array}{l}\text { No. of } \\
\text { hospitalizations } \\
\text { reviewed }\end{array}$} & \multicolumn{5}{|c|}{ No. of adverse events (and \% of hospitalizations reviewed) } \\
\hline & & Preventable & In-hospital* & Severe* & $\begin{array}{l}\text { Preventable } \\
\text { and severe }\end{array}$ & All \\
\hline \multicolumn{7}{|c|}{ Age quartile (and yr) } \\
\hline $1(<34)$ & 129 & $1 \quad(1)$ & $1(1)$ & 0 & 0 & $4 \quad(3)$ \\
\hline $2(34-54)$ & 127 & 0 & $6(5)$ & $1(1)$ & 0 & $13(10)$ \\
\hline $3(55-72)$ & 120 & $8 \quad(7)$ & $7(6)$ & $7(6)$ & $2(2)$ & $19(16)$ \\
\hline $4(>72)$ & 126 & $15(12)$ & $11(9)$ & $4(3)$ & $3(2)$ & $28(22)$ \\
\hline \multicolumn{7}{|c|}{ Admitting service } \\
\hline Medicine & 161 & $17(10)$ & $10(6)$ & $4(2)$ & $2(1)$ & $35(22)$ \\
\hline Surgery & 206 & $6 \quad(3)$ & $14(7)$ & $7(3)$ & $3(1)$ & $24(12)$ \\
\hline Ob/Gyn & 135 & $1 \quad(1)$ & $1(1)$ & $1(1)$ & 0 & $5 \quad(4)$ \\
\hline \multicolumn{7}{|c|}{ Admission status } \\
\hline Elective & 149 & $(2)$ & $8(5)$ & $2(1)$ & $1(1)$ & 12 (8) \\
\hline Emergency & 353 & $21 \quad(6)$ & $17(5)$ & $10(3)$ & $4(1)$ & $52(15)$ \\
\hline Entire sample & 502 & $24 \quad(5)$ & $25(5)$ & $12(2)$ & $5(1)$ & $64(13)$ \\
\hline
\end{tabular}

Note: $\mathrm{Ob} / \mathrm{Gyn}=$ obstetrics and gynecology

*Of these adverse events, $8(32 \%)$ of those in hospital and $5(42 \%)$ of those that were severe were preventable. 


\section{References}

1. Thomas EJ, Studdert DM, Burstin HR, Orav EJ, Zeena T, Williams EJ, et al. Incidence and types of adverse events and negligent care in Utah and Colorado. Med Care 2000;38:261-71.

2. Brennan TA, Leape LL, Laird NM, Hebert L, Localio AR, Lawthers AG, et al. Incidence of adverse events and negligence in hospitalized patients. Results of the Harvard Medical Practice Study I. N Engl 7 Med 1991;324:370-6.

3. Vincent C, Neale G, Woloshynowych M. Adverse events in British hospitals: preliminary retrospective record review. BM7 2001;322:517-9.

4. Wilson RM, Runciman WB, Gibberd RW, Harrison BT, Newby L, Hamilton JD. The Quality in Australian Health Care Study. Med 7 Aust 1995;163:458-71.

5. Thomas EJ, Studdert DM, Runciman WB, Webb RK, Sexton EJ, Wilson
RM, et al. A comparison of iatrogenic injury studies in Australia and the USA. I: Context, methods, casemix, population, patient and hospital characteristics. Int 7 Qual Health Care 2000;12:371-8.

6. Runciman WB, Webb RK, Helps SC, Thomas EJ, Sexton EJ, Studdert DM et al. A comparison of iatrogenic injury studies in Australia and the USA. II: Reviewer behaviour and quality of care. Int 7 Qual Health Care 2000;12:379-88.

7. Thomas EJ, Lipsitz SR, Studdert DM, Brennan TA. The reliability of medica record review for estimating adverse event rates. Ann Intern Med 2002;136:812-6.

Correspondence to: Dr. Alan J. Forster, Ottawa Hospital, Civic Campus, C406-1053 Carling Ave., Ottawa ON K1Y 4E9;

fax613 761-5351; aforster@ohri.ca

\section{Appendix 1: Chart-review methodology for identifying adverse events (AEs)}

\section{Screening criteria}

1. Unplanned admission as a result of health care management (including all readmissions)

2. Transfer from another acute care hospital

3. Transfer to the intensive care unit or another monitored setting

4. Unplanned transfer or return to the operating room

5. Unexpected death

6. Cardiorespiratory arrest

7. Unplanned removal, injury or repair of organ or structure during surgery, invasive procedure or vaginal delivery

8. Injury or complications related to abortion or labour and delivery, including neonatal complications

9. Hospital complications developing during admission (e.g., myocardial infarction, stroke or pulmonary embolism)

10. Development of neurologic deficit not present on admission (including change in level of consciousness or delirium)

11. Hospital-acquired infection

12. Hospital-incurred patient accident or injury

13. Adverse drug reaction indicated in the chart

14. Documentation of patient or family dissatisfaction with the care received

15. Documentation indicating litigation (contemplated or actual)

16. Any other undesirable outcome not covered by the other criteria

\section{Determination of adverse events by physicians}

A 6-point scale was used for rating whether an adverse outcome was due to treatment (definition of an AE)

1. No evidence that outcome was due to treatment

2. Little evidence that outcome was due to treatment

3. Outcome was possibly due to treatment (50/50 chance) but was more likely due to disease

4. Outcome was possibly due to treatment (50/50 chance) and was more likely due to treatment than to disease

5. Outcome was probably due to treatment

6. Outcome was definitely due to treatment

If the physician rating was 4 or higher, the chart was considered "AE-positive"; otherwise, it was "AE-negative." All AE-positive charts were reviewed again to minimize the chance of false-positive coding. In addition, for all AE-negative hospitalizations,

1 physician (T.R.A.) reviewed the case descriptions prepared by the nurse and initial physician-reviewer during the review process. Some of these descriptions indicated a high probability of an AE. For example, if the description stated that "pneumonia developed while the patient was in hospital," nosocomial infection, which should have been coded as an AE, was likely. All such "suspicious" hospitalizations were reviewed again.

\section{Reliability of physician reviews}

Of the 312 charts that were screen-positive, 58 were rated as AE-positive initially, and 50 of these were confirmed as AE-positive. Of the 254 charts rated as AE-negative initially, 36 had a suspicious case description; upon second review, the rating was changed to AE-positive for 14 . Thus, there were 8 false-positive, 14 false-negative and 22 true-negative charts among those reviewed twice.

\section{Results for charts that were reviewed twice by physicians}

First review; no. of charts

\begin{tabular}{lccc}
\cline { 2 - 4 } Second review & AE-positive & AE-negative & Total \\
\hline AE-positive & 50 & 14 & 64 \\
AE-negative & 8 & 22 & 30 \\
\hline Total & 58 & 36 & 94 \\
\hline
\end{tabular}

Note: The data indicate $77 \%$ agreement between reviewers; the kappa value for interrater reliability was moderate, at 0.5 ( $95 \%$ confidence interval $0.3-0.7)$. 


\section{Appendix 2: Case descriptions of AEs}

Case Description

Resulting in permanent disability or death and independently rated by 2 physician-reviewers as likely preventable

1 The patient, a known alcohol abuser, was admitted after a traumatic injury. Alcohol abuse was not diagnosed at the time of admission. Delirium tremens and myocardial infarction (MI) then developed.

2 The patient required amputation of a limb after a delay in diagnosis and transfer from a community hospital.

3 The patient was admitted after an intracerebral hemorrhage. Hypoxia developed; the diagnostic work-up for hypoxia was not complete. The patient was treated for presumed pneumonia. There was no clinical improvement for about 2 weeks, but no further investigations were ordered. Shock developed, and massive pulmonary embolism was diagnosed.

4 The patient was known to be at increased risk of thromboembolic disease. A pulmonary embolism developed days after orthopedic surgery. Postoperative anticoagulant therapy was not adequate.

5 Diarrhea associated with Clostridium difficile toxin developed after antibiotic treatment of pneumonia and was complicated by dehydration and acute renal failure.

\section{Independently rated by 2 physician-reviewers as likely preventable}

6 Toxicity to digoxin therapy developed, with electrolyte imbalances and neurologic symptoms.

7 Delirium developed while the patient was receiving narcotic and antipsychotic medications.

8 Falls occurred secondary to medication use.

9 After a surgical procedure, the patient had respiratory depression secondary to narcotic, sedative and antiemetic medication. Subsequently, delirium developed.

10 Acute renal failure was caused by diuretic and angiotensin-converting-enzyme inhibitor.

11 The patient was given an excessive dose of anticoagulant medication and subsequently had serious gastrointestinal (Gl) tract hemorrhage.

12 The patient, known to have diabetes mellitus, was admitted with pneumonia and poorly controlled blood glucose concentration, then readmitted 48 hours after discharge because of continued elevation in the blood glucose concentration.

13 Urinary tract infection developed secondary to unnecessary use of a urinary bladder catheter.

14 The patient, known to be taking anticoagulants, presented to the emergency department with massive epistaxis. Laboratory investigation revealed therapeutic anticoagulation and a reduction in the hemoglobin level of $2 \mathrm{~g} / \mathrm{L}$ during the patient's stay in the emergency department. After discharge with local therapy but no reversal of anticoagulation, the patient required admission within 24 hours because of continued hemorrhage.

15 Upper Gl tract hemorrhage developed because of a gastric ulcer that was secondary to long-term inappropriate use of ASA.

16 During a recent hospitalization the patient's insulin was discontinued. The patient was readmitted in a hyperosmolar nonketotic state.

17 Toxicity to digoxin therapy developed.

18 The patient was known to be allergic to penicillin but was given a cephalosporin for an infection. Generalized rash and swelling developed.

19 The patient was admitted because of complications of metastatic cancer. On the day of discharge, the patient required readmission because of continued confusion and pain.

20 The patient, known to have renal insufficiency, was seen in the emergency department with a urinary tract infection and discharged without laboratory assessment of renal function. The patient was admitted 1 week later with acute renal failure.

21 The patient became confused while taking several medications prescribed for pain and required hospital admission for management.

22 During a 2-day wait for an operative procedure, the patient fasted and subsequently became delirious owing to dehydration.

23 The patient became confused while taking many medications for pain and required hospital admission for management.

24 After discharge home from the emergency department with a diagnosis of hyperkalemia and hyperglycemia, the patient required hospital admission within 24 hours because of hyperglycemia.

\section{Resulting in permanent disability or death and likely not preventable}

25 A wound infection developed after surgery.

26 A serious infection and neutropenia developed 2 weeks after chemotherapy in a patient with advanced cancer. The patient died 3 days after admission.

27 The patient was admitted for surgical repair of enterocutaneous fistulas caused by radiation treatment.

28 Multiple postoperative complications, including pneumonia, resulted in death.

29 The patient had difficulty swallowing after chemotherapy and radiation therapy for cancer.

30 The patient had an $\mathrm{MI}$ and received thrombolytic therapy. A fatal intracerebral hemorrhage occurred.

31 Infection occurred in a wound from a previous surgical procedure.

\section{Remaining AEs}

32 The patient underwent vascular surgery, then required a second operation 1 week later because the first procedure failed.

33 The patient, who had metastatic cancer, was admitted to hospital for staging investigations. Pneumonia developed during the hospital stay.

34 Febrile neutropenia developed after chemotherapy.

35 C. difficile colitis developed after antibiotic treatment. Subsequently, the patient had life-threatening complications from the colitis.

36 The patient underwent a surgical procedure. After engaging in inappropriate activity, the patient was readmitted 1 day after discharge and subsequently required a second surgical procedure. 


\section{Appendix 2 continued}

\section{Remaining AEs continued}

37 Chemotherapy for cancer caused depression of the patient's immune system and an infection developed.

38 The patient was admitted for percutaneous drainage of an infected gallbladder and, 2 weeks later, was readmitted because of acute renal failure and sepsis secondary to blockage of the drain.

39 The patient experienced complications of ascites, nausea and vomiting after hormonal therapy and required percutaneous drainage.

40 A patient with drainage complications from a nephrostomy tube was admitted 1 day after discharge because of continued complications.

41 Infection of a dialysis catheter necessitated admission and antibiotic treatment.

42 The patient had a massive hemorrhage after a biopsy procedure.

43 Pneumonia and febrile neutropenia developed after chemotherapy.

44 While the patient was being treated for severe injuries from a motor vehicle crash, nosocomial pneumonia developed.

45 Acute renal failure, antibiotic-associated diarrhea and falls developed.

46 Acute renal failure developed secondary to diuretic use.

$47 \quad$ Nosocomial pneumonia developed.

48 Massive hemorrhage developed during surgery because of laceration of a blood vessel.

49 MI developed after a vascular operative procedure.

50 Wound dehiscence developed after a surgical procedure.

51 Small-bowel obstruction developed 1 week after bowel surgery.

52 Hypoventilation and hypoxia developed during prescribed narcotic analgesia after surgery.

53 The patient had incomplete skin healing after surgery and required plastic surgery.

54 The patient underwent a planned minimally invasive operation and, 1 week later, an operation for the same indication that was not minimally invasive.

55 Postoperative bleeding necessitated readmission.

56 Febrile neutropenia developed during chemotherapy for cancer.

57 After antibiotic therapy for a serious infection, diarrhea associated with $C$. difficile developed.

58 Syncope developed after an increase in the dosage of topical nitrate therapy.

59 Postoperative pneumonia necessitated admission to hospital.

60 Abdominal pain and diarrhea associated with the $C$. difficile toxin developed after antibiotic treatment of a urinary tract infection.

61 Nausea and constipation developed during chemotherapy for cancer.

62 Admission was required because of a wound infection 2 weeks after gynecologic surgery.

63 Recurrent infectious complications from a previous surgical complication necessitated admission and antibiotic treatment.

64 Upper Gl tract bleeding, which necessitated admission, was deemed to be secondary to prescribed ASA. 